\section{Case Reports in Neurology}

Case Rep Neurol 2021;13:100-107

DOI: $10.1159 / 00051212$

Published online: February 15, 2021

(C) 2021 The Author(s)

Published by S. Karger AG, Base

www.karger.com/crn

This article is licensed under the Creative Commons Attribution-NonCommercial 4.0 International License (CC BY-NC) (http://www.karger.com/Services/OpenAccessLicense).

Usage and distribution for commercial purposes requires written permission.

\title{
A 56-Year-Old Woman with Recurrent Strokes: A Clear Case with a Therapeutic Dilemma
}

\author{
Eric S. Miller ${ }^{a}$ Haitham M. Hussein ${ }^{b} \quad$ Elie Gertner ${ }^{a}$ \\ a Section of Rheumatology, Regions Hospital, St. Paul, Minnesota and Division of \\ Rheumatology, Minneapolis, MN, USA; bSection of Neurology, Regions Hospital \\ Comprehensive Stroke Center, Regions Hospital and Division, St. Paul, MN, USA
}

\section{Keywords}

Antiphospholipid syndrome $\cdot$ Ischemic stroke $\cdot$ Cerebral amyloid angiopathy $\cdot$ Hemorrhagic stroke $\cdot$ Stroke

\begin{abstract}
A 56-year-old woman with a history of cerebral amyloid angiopathy (CAA) complicated by prior intracranial hemorrhage $(\mathrm{ICH})$ was evaluated for an asymptomatic ischemic stroke discovered on screening brain MRI. On echocardiogram, she was found to have a mass on her mitral valve and strongly positive antiphospholipid antibodies. She was diagnosed with nonbacterial thrombotic (Libman-Sacks) endocarditis associated with the primary antiphospholipid syndrome (APS). The treatment decision was complicated by the history of CAA with ICH within the last year with very high risk for bleeding complications if on anticoagulation. A multidisciplinary decision was made to initiate a trial of warfarin for 3 months. She fared well and warfarin was continued. She has not had any further bleeding or ischemic events over the subsequent 1.5 years and remains on warfarin for her APS.

(C) 2021 The Author(s)

Published by S. Karger AG, Basel
\end{abstract}

\section{Introduction}

Patients with both cerebral amyloid angiopathy (CAA) and antiphospholipid antibody syndrome (APS) pose a therapeutic challenge for clinicians due to their inherent risks of

\begin{tabular}{ll}
\hline & Eric S. Miller \\
Regions Hospital, Section of Rheumatology \\
640 Jackson Street, Mail Stop 11107E \\
St. Paul, MN 55101 (USA) \\
mill3558@umn.edu
\end{tabular}




\section{Case Reports in Neurology}

Case Rep Neurol 2021;13:100-107

DOI: 10.1159/000512126

(c) 2021 The Author(s). Published by S. Karger AG, Basel www.karger.com/crn

Miller et al.: A 56-Year-Old Woman with Recurrent Strokes: A Clear Case with a Therapeutic Dilemma

hemorrhage and ischemic strokes, respectively. Decisions to start anticoagulation should be based on personalized risk and benefits of the conditions and treatment. Clinical and radiologic monitoring are important during treatment of concurrent CAA and APS. Further studies are needed to evaluate the safety of anticoagulation in patients with CAA.

\section{Case Report}

A 56-year-old Caucasian woman with a history of hypertension and migraine headaches was found by her husband walking in circles and acting strangely. She was last acting normally the night before and there were no signs of trauma. She was evaluated in an emergency department and found to have an intracerebral hemorrhage (ICH) in the left frontal cortex on head CT (Fig. 1). She had a history of mild hypertension but had not been on antihypertensive therapy.

She had not taken any anti-thrombotic medications prior to the ICH except for occasional aspirin-containing migraine therapy. Her coagulation profile was normal except for elevated activated partial thrombin time (aPTT). CT angiogram and MR angiogram did not detect any abnormal blood vessels. MRI of the brain did not show an underlying tumor or infarction. She was discharged on a low-dose antihypertensive agent. A repeat MRI of the brain was done a month later (Fig. 2) and showed 9 microhemorrhages and superficial siderosis meeting the Boston criteria for probable CAA [1]. Five months after the ICH, a repeat brain MRI showed a new area of encephalomalacia in the left occipital cortex that was not present on the prior MRI (Fig. 3). The radiological features indicated a chronic infarction [1]. The MRI also showed an area of encephalomalacia of the right frontal cortex/subcortex that had been seen on the initial CT and MRI but had unclear significance. With the development of the left occipital encephalomalacia, it became apparent that the right frontal lesion was also a chronic infarction. A transthoracic echocardiogram was done as part of the ischemic stroke investigations and showed an echogenic structure on the atrial aspect of the anterior mitral leaflet. Transesophageal echocardiogram was done on the same day and showed a 6-mm, sessile, thickened mass on the mitral valve (Fig. 4). She was admitted to the hospital for expedited workup. She was asymptomatic, did not show any systemic manifestations of infective endocarditis, and had normal vital signs. Her laboratory tests were significant for mild leukopenia, thrombocytopenia, and mild renal insufficiency. She also was noted to have aPTT of $54 \mathrm{~s}$ (normal: 22.5-36.5 s). Further hypercoagulable workup revealed positive lupus anticoagulant (LA), high-titer anticardiolipin (aCL) IgG antibody, and high-titer beta-2-glycoprotein 1 IgG antibody (aB2GP1) suggestive of APS. Additional autoimmune evaluation revealed low-titer ANA 1:40 and hypocomplementemia. The patient was diagnosed with nonbacterial thrombotic (Libman-Sacks) endocarditis due to primary APS. She did not meet criteria for another connective tissue disease. After multidisciplinary discussion regarding her underlying CAA and recent ICH, anticoagulation with warfarin was recommended for at least 3 months with INR goal of 2-3.

After 3 months on warfarin with INR documented between 1.6-2.6, a follow-up transthoracic echocardiogram showed a slightly smaller mass on the mitral valve. Anticoagulation was continued due to the persistent mass, as well as lack of adverse events. Repeat testing at that time for antiphospholipid antibodies (aPL) showed they were present in high titer confirming the diagnosis of APS. Indefinite anticoagulation was recommended.

In the one and half years since her initiation on warfarin, our patient has not had any major bleeding or thrombotic events, including intracranial bleeding. She has mild cognitive

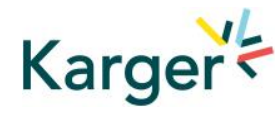




\section{Case Reports in Neurology}

Case Rep Neurol 2021;13:100-107

DOI: 10.1159/000512126

(c) 2021 The Author(s). Published by S. Karger AG, Basel www.karger.com/crn

Miller et al.: A 56-Year-Old Woman with Recurrent Strokes: A Clear Case with a Therapeutic Dilemma

impairment without focal findings. Her most recent brain MRI, completed 18 months after the ICH, showed no new areas of ischemia or lobar hemorrhages, although there were 4 new microhemorrhages compared to the prior study (Fig. 5). No symptoms of another collagen vascular disease have developed.

\section{Discussion}

CAA is a condition characterized by amyloid deposition primarily in the walls of central nervous system arteries, which leads to fragile blood vessels that are prone to rupture. It is usually a sporadic condition that occurs in elderly individuals but a hereditary form can present earlier in life and cause more severe manifestations [2]. Distribution of CAA lesions are classically in the periphery of occipital, frontal, temporal, and parietal lobes. This is in contrast to those hemorrhagic lesions seen secondary to hypertension, which tend to be more central and involving the deep brain structures $[3,4]$. Clinically, CAA can present as sporadic lobar hemorrhages causing altered mental status, focal neurologic deficits, coma, or death. However, they may also present with asymptomatic microbleeds leading to progressive cognitive decline and dementia [2]. Patients with CAA have an average ICH recurrence rate of approximately $9 \%$ per year, though the specific risk varies depending on their CAA biomarkers $[5,6]$. Clinical diagnosis can be difficult and a definitive diagnosis is only made with tissue biopsy. The Boston criteria for CAA diagnosis allow clinicians to make a probable diagnosis based on clinical presentation, age, and exclusion of alternative etiologies for hemorrhage [2]. Unfortunately, there is no treatment of CAA and at the present time, the goal is to minimize the risk of recurrent intracranial bleeding. This includes ensuring adequate blood pressure control and avoiding anticoagulation. Our patient meets the Boston diagnostic criteria for probable CAA. Although she had been diagnosed with mild hypertension and had not been on an antihypertensive agent prior to her ICH, CAA is probably the underlying pathology given the cortical location of the left frontal hemorrhage, the microhemorrhages, and the sparing of the deep grey brain tissue such as basal ganglia, thalamus, and brainstem.

APS is an autoimmune disease characterized by thrombosis and obstetrical events in the presence of aPL. Diagnosis involves the occurrence of a thrombotic event and the laboratory presence of aPL. The three most commonly tested autoantibodies include LA, B2GP1 antibodies, and aCL antibodies [7]. The diagnosis of APS requires at least one aPL to be present in a moderate-to-high titer with a repeat test at least 12 weeks later to confirm. Patients that are positive for all three aPL are considered "triple positive" and thought to be at the highest risk of events $[7,8]$. In a cohort of triple positive APS patients the thrombotic event rate after 10 years was $44.2 \%$. Anticoagulation significantly decreased the rate of recurrent thrombosis in these patients [7]. The cornerstone of treatment for APS is anticoagulation with a vitamin $\mathrm{K}$ antagonist and those with high-risk profiles are recommended to remain on anticoagulation indefinitely. The need for anticoagulation is even greater when a patient, such as the one described, has Libman-Sacks endocarditis, which itself is an independent risk factor for embolic phenomena [9].

This patient represents a clinical challenge. There are no clinical trials regarding use of anticoagulation for APS in patients with CAA, especially those with history of ICH. Guidelines for management of non-valvular atrial fibrillation in patients with CAA suggests stratifying the ischemic risk against hemorrhagic risk. Generally, if a patient has CAA, anticoagulation is usually associated with higher risk of ICH. However, if the clinician feels that the risk of ischemic

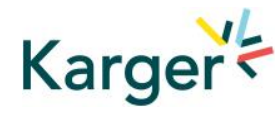




\section{Case Reports in Neurology}

Case Rep Neurol 2021;13:100-107

DOI: 10.1159/000512126

(c) 2021 The Author(s). Published by S. Karger AG, Basel www.karger.com/crn

Miller et al:: A 56-Year-Old Woman with Recurrent Strokes: A Clear Case with a Therapeutic Dilemma

stroke outweighs the risk of hemorrhage, then oral anticoagulation is favored [6]. The radiological finding of superficial siderosis was associated with the highest risk of recurrent ICH [6]. A meta-analysis evaluating the use of oral anticoagulants in patients with ICH found that resuming oral anticoagulants led to favorable outcomes after 1 year, including those categorized as lobar ICH related to CAA [10]. In our case, the decision to anti-coagulate was made based on our assessment that the presence of the mitral valve vegetation along with MRI finding suggestive of an embolic stroke was the higher risk condition compared to CAA. We also envisioned that better control of hypertension would provide more protection from recurrent ICH. Our patient had only one symptomatic ICH 5 months earlier and only trace superficial siderosis. The detection of another MRI lesion consistent with a silent ischemic stroke that was older than the ICH was also interpreted as evidence of higher ischemic risk. As well, brain MRI before initiation of anticoagulation did not show progression of CAA compared to the one done at the time of ICH. Repeat MRI 1 year after anticoagulation only showed a few microhemorrhages that had not been seen before.

In a patient with the rare association of CAA and APS, without randomized controlled trials, clinicians must individualize treatment based on the patient's overall thrombotic and hemorrhagic risk. Fortunately, our patient has done well with anticoagulation and has remained free of ischemic hemorrhage or ICH. We plan on continuing anticoagulation and monitoring the patient clinically and radiologically with MRI. If there is evidence of significant progression of CAA, which is expected with age, or any recurrent ICH, the therapeutic plan may need to change.

\section{Conclusion}

In patients with symptomatic CAA and high-risk APS, the decision to start anticoagulation is challenging. In the absence of clinical guidelines, multidisciplinary decision-making and personalized management based upon the risks and benefits of the conditions and the treatment are necessary to make the safest therapeutic choice.

\section{Statement of Ethics}

Patient is not identified by name in this paper. She has reviewed the manuscript in entirety, including MRI/CT imaging, and has approved its contents for accuracy. She has provided written informed consent for publication of this case report and any accompanying images. This case report did not require IRB approval.

\section{Conflict of Interest Statement}

Authors have no conflicts of interest.

\section{Funding Sources}

None.

\section{Karger'}




\section{Case Reports in Neurology}

\begin{tabular}{l|l}
\hline Case Rep Neurol 2021;13:100-107 \\
\hline DOI: 10.1159/000512126 & $\begin{array}{l}\text { ○ 2021 The Author(s). Published by S. Karger AG, Basel } \\
\text { www.karger.com/crn }\end{array}$ \\
\hline
\end{tabular}

Miller et al.: A 56-Year-Old Woman with Recurrent Strokes: A Clear Case with a Therapeutic Dilemma

\section{Author Contributions}

All authors listed have contributed to drafting, writing, and editing of the manuscript. Eric S. Miller: draft, writing, editing, submission. Haitham M. Hussein: editing, writing, figures. Elie Gertner: editing, final review.

\section{References}

1 Price T, Manolio T, Kronmal R, Kittner S, Yue N, Robbins J, et al. Silent Brain Infarction on Magnetic Resonace Imaging and Neurological Abnormalities in Community-Dwelling Older Adults. Stroke. 1997;28(6):1158-64.

2 Biffi A, Greenberg SM. Cerebral amyloid angiopathy: a systematic review. J Clin Neurol. 2011 Mar;7(1):1-9.

3 Pasi M, Marini S, Morotti A, Boulouis G, Xiong L, Charidimou A, et al. Cerebellar Hematoma Location: Implications for the Underlying Microangiopathy. Stroke. 2018 Jan;49(1):207-10.

4 Rosand J, Muzikansky A, Kumar A, Wisco JJ, Smith EE, Betensky RA, et al. Spatial clustering of hemorrhages in probable cerebral amyloid angiopathy. Ann Neurol. 2005 Sep;58(3):459-62.

5 Banerjee G, Carare R, Cordonnier C, Greenberg SM, Schneider JA, Smith EE, et al. The increasing impact of cerebral amyloid angiopathy: essential new insights for clinical practice. J Neurol Neurosurg Psychiatry. 2017 Nov;88(11):982-94.

6 Cannistraro RJ, Meschia JF. The Clinical Dilemma of Anticoagulation Use in Patients with Cerebral Amyloid Angiopathy and Atrial Fibrillation. Curr Cardiol Rep. 2018 Sep;20(11):106.

7 Pengo V, Ruffatti A, Legnani C, Gresele P, Barcellona D, Erba N, et al. Clinical course of high-risk patients diagnosed with antiphospholipid syndrome. J Thromb Haemost. 2010 Feb;8(2):237-42.

8 Garcia D, Erkan D. Diagnosis and Management of the Antiphospholipid Syndrome. N Engl J Med. 2018 May;378(21):2010-21.

9 Lee JL, Naguwa SM, Cheema GS, Gershwin ME. Revisiting Libman-Sacks endocarditis: a historical review and update. Clin Rev Allergy Immunol. 2009 Jun;36(2-3):126-30.

10 Biffi A, Kuramatsu JB, Leasure A, Kamel H, Kourkoulis C, Schwab K, et al. Oral Anticoagulation and Functional Outcome after Intracerebral Hemorrhage. Ann Neurol. 2017 Nov;82(5):755-65.

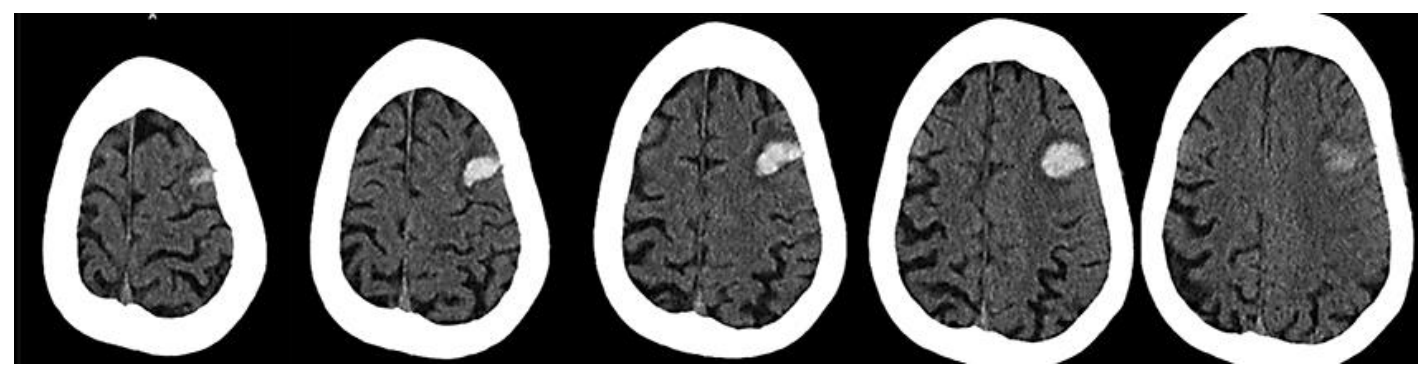

Fig. 1. Initial CT scan of the head showing left frontal cortical intracerebral hemorrhage measuring $5.7 \mathrm{~mL}$ with surrounding vasogenic edema. 


\section{Case Reports in Neurology}

\begin{tabular}{l|l}
\hline Case Rep Neurol 2021;13:100-107 \\
\hline DOI: 10.1159/000512126 & $\begin{array}{l}\text { C 2 2021 The Author(s). Published by S. Karger AG, Basel } \\
\text { www.karger.com/crn }\end{array}$ \\
\hline
\end{tabular}
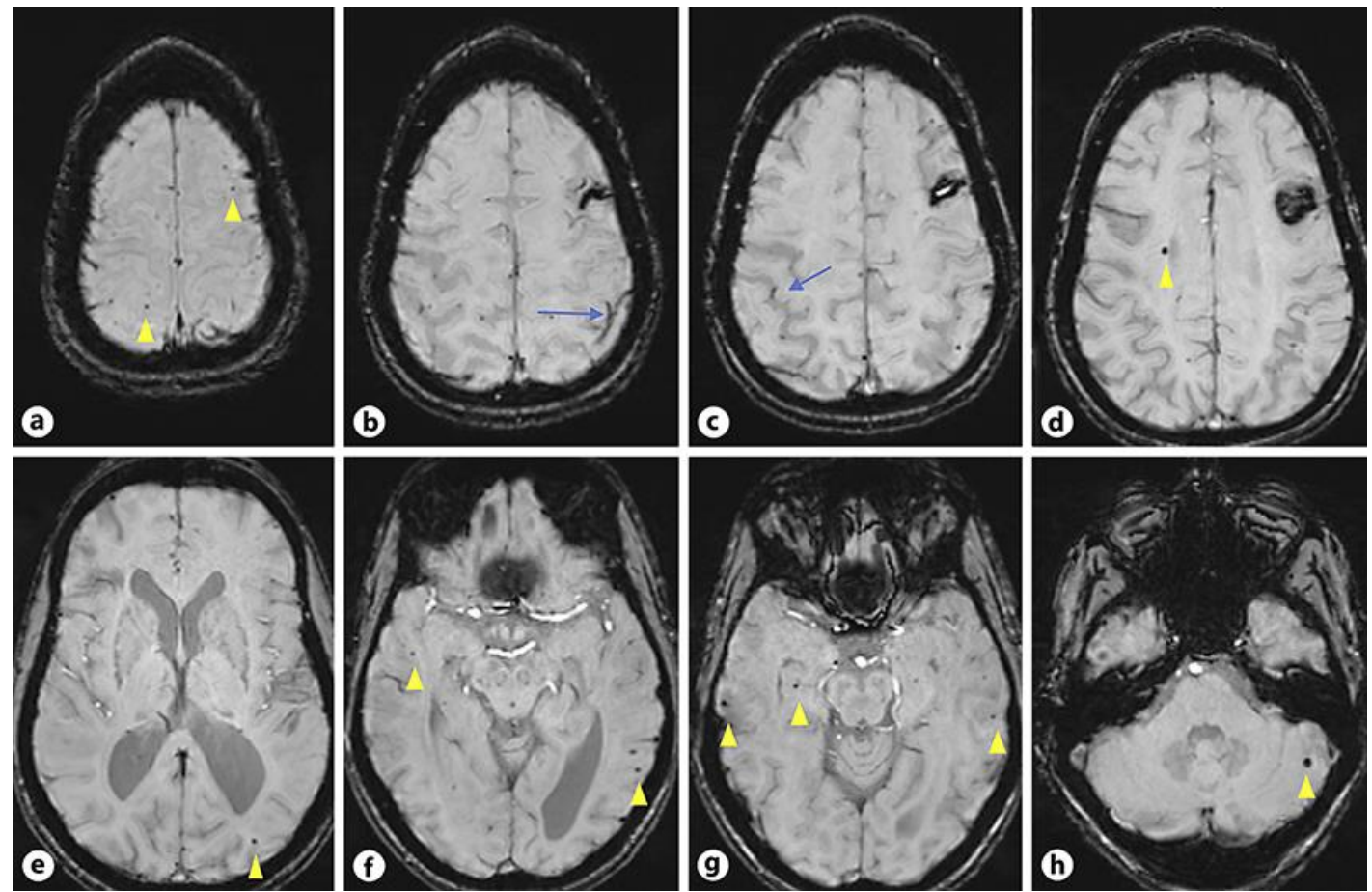

Fig. 2. Susceptibility-weighted imaging sequence of a brain MRI done 1 month after intracerebral hemorrhage showing the left frontal lobar hemorrhage (b-d), multiple microhemorrhages (yellow arrowhead) in the cortical and subcortical areas but not the deep grey matter, and superficial siderosis (blue arrow). 


\section{Case Reports in Neurology}
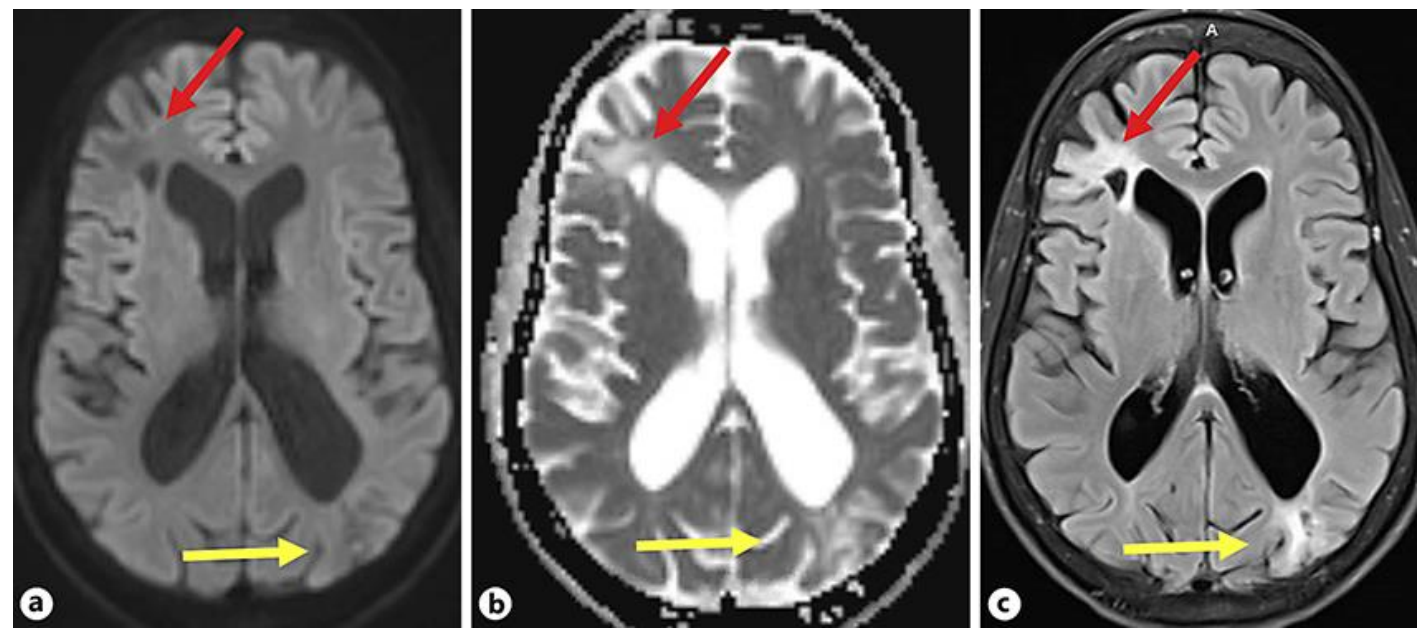

Fig. 3. Diffusion-weighted imaging (a), apparent diffusion coefficient (b) map, and fluid attenuated inversion recovery (c) sequences of an MRI done 5 months after the intracerebral hemorrhage showing the development of a left occipital encephalomalacia (yellow arrow) that was new compared to the prior MRI done 3 months earlier representing a chronic infarction. The images also show a right frontal cortical encephalomalacia that had been seen from on initial imaging and was of unclear significance until the left occipital encephalomalacia developed. At that point, the impression was that the right frontal encephalomalacia was probably a chronic infarction too.

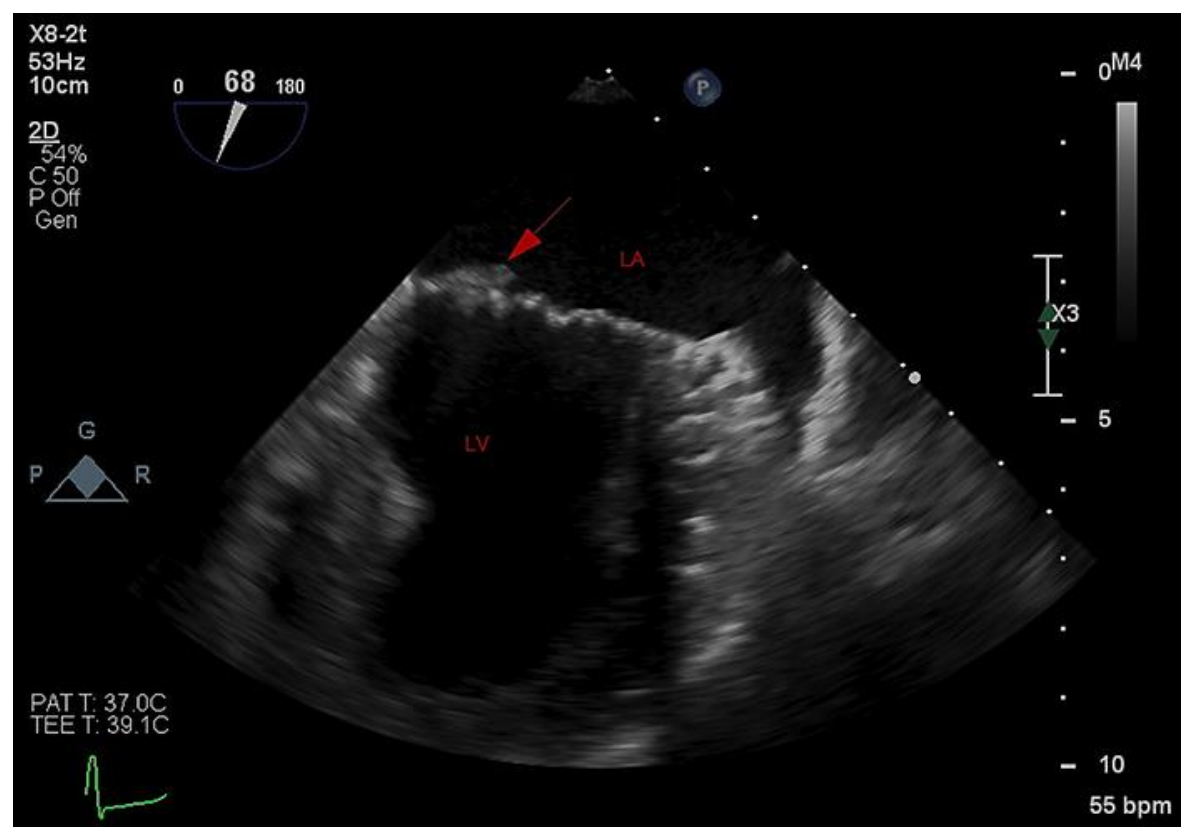

Fig. 4. Transesophageal echocardiogram image showing a 6-mm, sessile, thickened mass (red arrowhead) on mitral valve. Left atrium (LA) and left ventricle (LV) are labeled. 


\section{Case Reports in Neurology}

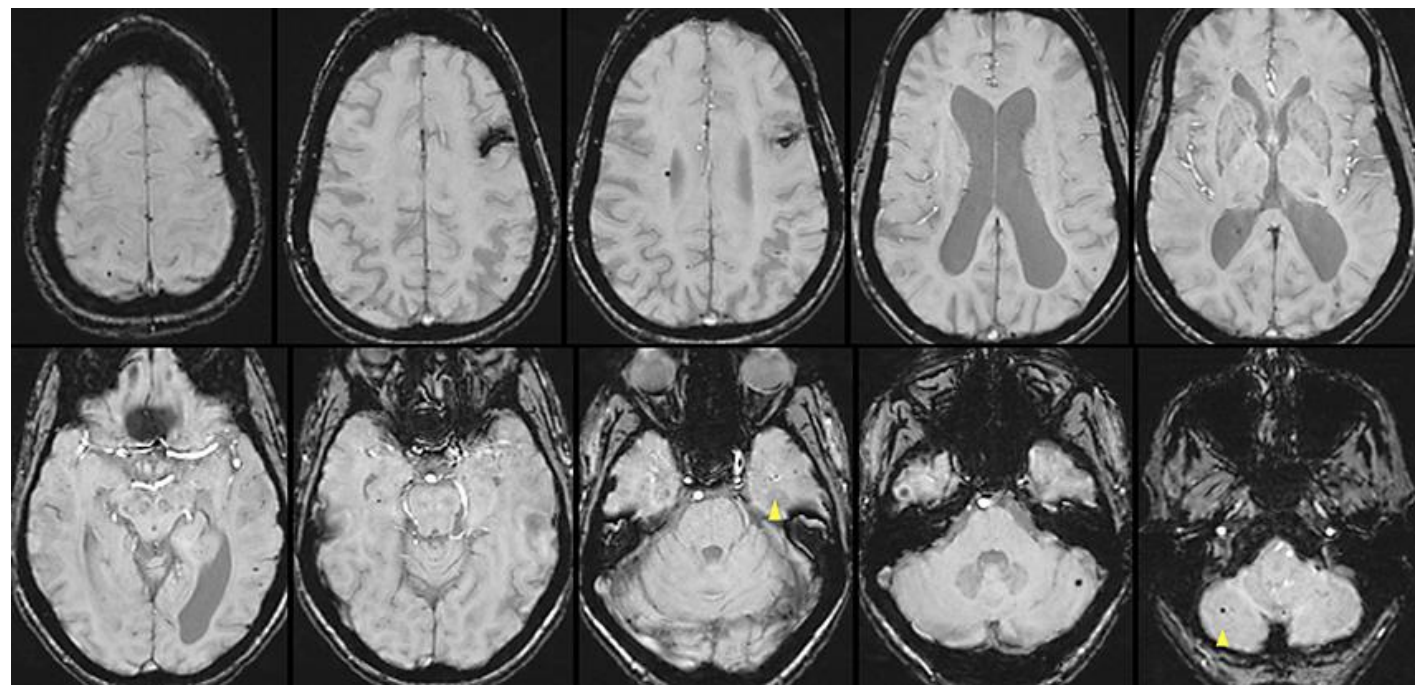

Fig. 5. Susceptibility-weighted imaging sequence of a brain MRI done 18 months after the left frontal intracerebral hemorrhage (ICH) showed no new lobar hemorrhages although a few new microhemorrhages (yellow arrowhead) could be detected compared to the MRI done 1 month after the ICH. 